\title{
OBSERVATION OF INTERFERENCE FRINGES IN BRAGG-CASE SYNCHROTRON DOUBLE-CRYSTAL IMAGES OF STACKING FAULTS IN DIAMOND
}

\author{
W. WIERZCIIOWSKI \\ Institute of Electronic Materials Technology \\ Wólczyńska 133, 01-919 Warsaw, Poland \\ AND M. MOORE \\ Department of Physics, Royal Holloway and Bedford New College \\ University of London \\ Egham, Surrey, TW20 0EX, Great Britain
}

\begin{abstract}
Bragg-case synchrotron double-crystal images of stacking faults were studied in a slab prepared from a synthetic diamond of a good quality. The images of stacking faults in topographs taken on the tails of the rocking curve exhibited well pronounced interference fringes. The fringes were strongly dependent on the angular setting and they were less spaced further from the maximum. The experimental images were compared with those theoretically predicted from an application of plane-wave dynamical theory. A reasonably good correspondence between theoretical and experimental images was obtained. The theoretical images of stacking faults were dependent on the type of stacking fault, producing some difference in the first fringe.
\end{abstract}

PACS numbers: $61.10 .-\mathrm{i}, 61.70 .-\mathrm{r}$

\section{Introduction}

Most X-ray investigations of stacking faults have used transmission section topography and spherical-wave theory [1-5]. The observation of interference fringes is more difficult in the back-reflection diffraction geometry and to our best knowledge no observation of fringe patterns in stacking fault images have been reported in that case.

In the present experiment we obtained interference fringes in Bragg-case synchrotron double-crystal topographs of stacking faults in diamond. Both low absorption of $\mathrm{X}$-rays and high collimation of the beam was important for the visibility of the fringes. Stacking faults can be easily found in otherwise good quality diamonds obtained using the reconstitution method [6]. 


\section{Theoretical}

We concentrate here on the Bragg-Laue case of stacking fault images which provided better fringe patterns in the present experiment. The position of wave points in this case is shown in Fig. 1. The wave field excited in the first region of the crystal is split by the stacking fault into reflected and transmitted beams. Behind the fault plane these two beams excite four new wave fields corresponding to two wave points only, both situated on the normal to the fault plane. One of these points has the same real coordinates as the wave point of the field induced in the first region. The fifth wave field of the same coordinates as in region $I$ is induced by the incident wave.

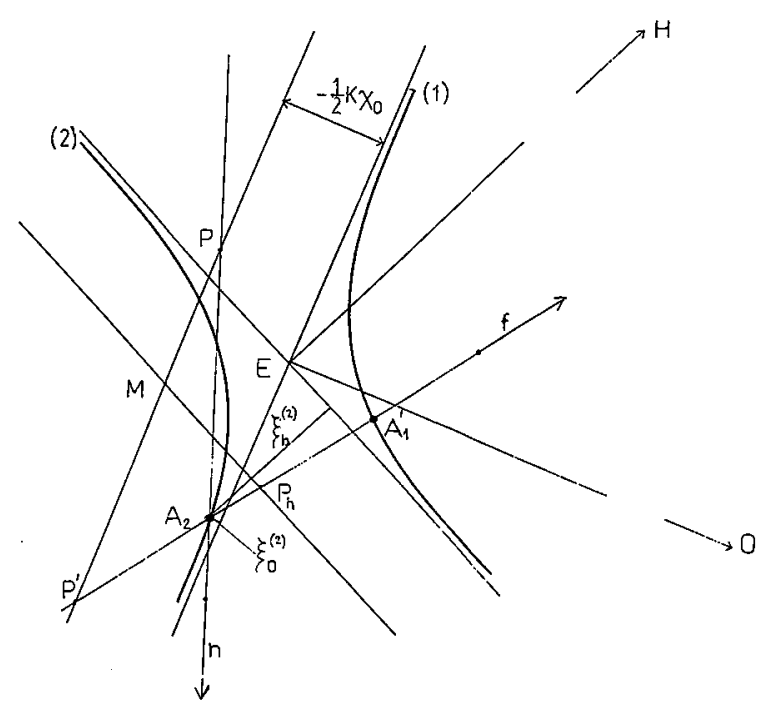

Fig. 1. Positions of the wave points on the dispersion surfaces. Situations correspond to the low-angle tail of rocking curve. The single wave point excited primarily in the first region $P_{2}$ is located on the normal to the entrance surface. The Poynting vector connected with this point is directed inwards towards the crystal. One of the points excited in the second part of the crystal coincides with this point, while the second point $P_{1}^{\prime}$ is located on the second branch of the dispersion surface on the normal to the fault plane passing through $P_{2}$.

The formulas giving the coordinates $\xi_{0 i}, \xi_{h i}$ and the amplitudes $D_{0 i}, D_{h i}$ of the wave field induced in the region I can be found in many papers (see e.g. [7]). This wave field is of type II for low angles of incidence $\Re y>-1$ and of type I for $\Re y<-1$. The angular variable $y$ is here

$$
y=-\frac{2 \sin 2 \Theta \Delta \Theta+\chi_{0}\left(1-\gamma_{h} / \gamma_{0}\right)}{2|C| \sqrt{\left|\gamma_{h}\right| / \gamma_{0}} \sqrt{\chi_{h} \chi_{\bar{h}}}}
$$

where $\Delta \Theta$ is the deviation from the Bragg angle $\Theta, \chi_{0}, \chi_{h}, \chi_{\bar{h}}$ are the Fourier coefficients of dielectric susceptibility, $C$ is the polarization factor and $\gamma_{0}, \gamma_{h}$ are 
the direction cosines of the transmitted and reflected beams with respect to the normal to the entrance surface.

As the second part of the crystal is shifted with respect to the first, by the fault vector $f$, the Fourier coefficients of dielectric susceptibility in region II are

$$
\chi_{h}^{\prime}=\chi_{h} \exp (2 \pi \mathrm{i} h \cdot f), \quad \chi_{\bar{h}}^{\prime}=\chi_{\bar{h}} \exp (-2 \pi \mathrm{i} h \cdot f),
$$

where $h$ is considered reciprocal vector.

To find the wave points with the correct imaginary parts we found the deviations from the Bragg angle of the beams created by the decomposition of the wave field in the fault plane treated formally as vacuum waves. By some geometrical considerations which may be found from Fig. 1, these deviations are for the beam in the transmitted direction:

$$
\Delta \Theta_{0 i}^{\prime}=\Delta \Theta+\Re\left(-\xi_{0}^{(i)}-\frac{1}{2} \chi_{0} K\right) \frac{\sin \alpha}{\gamma_{0} \gamma_{0}^{\prime}},
$$

and for the beam in the reflected direction:

$$
\Delta \Theta_{h i}^{\prime}=-\frac{\gamma_{0}^{\prime}}{\gamma_{h}^{\prime}} \Delta \Theta_{0 i}^{\prime}
$$

where $\gamma_{0}^{\prime}$ and $\gamma_{h}^{\prime}$ are now the direction cosines with respect to the normal to the fault plane, $K$ is the wave number of the incident wave. We use these new deviations for calculation the new wave points having the same real coordinates and the imaginary parts corresponding to the normal $f$. The remaining coordinates of the wave points excited by the transmitted beam are

$$
\begin{aligned}
& \xi_{h i(0 i)}=\frac{\chi_{h}^{\prime} \chi_{\bar{h}^{\prime}} C^{2}}{4 \xi_{0 i(0 i)}}, \\
& \xi_{0 j(0 i)}=-\frac{\gamma_{0}^{\prime}}{\gamma_{h}^{\prime}} \xi_{h i(0 i)}, \\
& \xi_{h j(0 i)}=-\frac{\gamma_{h}^{\prime}}{\gamma_{0}^{\prime}} \xi_{0 i(0 i)} ;
\end{aligned}
$$

$j=1,2, i \neq j$ and the indices of the incident wave (in brackets) are $i=1$ for $\Re y<-1$ and $i=2$ for $\Re y>1$. The present theory is not considered in the total reflection range $1>\Re y>-1$.

The coordinates of the wave points induced by the reflected beam can now be found similarly by noticing that the reflected beam may be treated as the incident beam for the $-h$ diffraction vector with the deviation from the Bragg angle $\Delta \Theta_{h i}^{\prime}$.

The amplitudes of the waves induced by the transmission beam are

$$
\begin{aligned}
& D_{h 1(0 i)}=D_{0 i} \frac{2}{K \chi_{\bar{h}}^{\prime} C} \frac{\xi_{01(0 i)} \xi_{02(0 i)}}{\xi_{02(0 i)}-\xi_{01(0 i)}}, \\
& D_{h 2(0 i)}=-D_{h 1(0 i)},
\end{aligned}
$$

and those excited by the reflected beam

$$
\begin{aligned}
& D_{h 1(h i)}=D_{h i(h i)} \frac{\xi_{h 2(h i)}}{\xi_{h 2(h i)}-\xi_{h 1(h i)}}, \\
& D_{h 2(h i)}=-D_{h 1} .
\end{aligned}
$$


The amplitude of the wave field excited by the incident wave in region II has different phase than in region $I$,

$$
D_{h 1(a)}=D_{h i} \exp (2 \pi \mathrm{i} h \cdot f) \text {. }
$$

The resultant amplitude of the reflected beam may be written for $\Re y>1$ as a function of $x$, the distance from the stacking fault outcrop along the surface may be written

$$
\begin{aligned}
& D_{h}^{(a)}(x)=\left[D_{h 1(02)} \exp \left(-2 \pi \mathrm{i} \Im k_{h 1(02)} x \sin \alpha\right)+D_{h 1(h 2)}\right. \\
& \left.\times \exp \left(-2 \pi \mathrm{i} \Im k_{h 1(02)} x \sin \alpha\right)\right] \exp \left(-2 \pi \mathrm{i} \Im k_{02} x \frac{\gamma_{h}}{\gamma_{h}^{\prime}} \sin \alpha\right) \exp \left(2 \pi \mathrm{i} \Re k_{01(02)} x \gamma_{h}\right) \\
& +\left\{\left[D_{h 2(02)} \exp \left(-2 \pi \mathrm{i} \Im k_{h 2(02)} x \sin \alpha\right)+D_{h 2(h 2)} \exp \left(-2 \pi \mathrm{i} \Im k_{h 2(02)} x \sin \alpha\right)\right]\right. \\
& \left.\times \exp \left(-2 \pi \mathrm{i} \Im k_{02} x \frac{\gamma_{h}}{\gamma_{h}^{\prime}} \sin \alpha\right)+D_{h 2(a)}\right\} \exp \left(2 \pi \mathrm{i} \Re k_{02(02)} x \gamma_{h}\right)
\end{aligned}
$$

and for $\Re y<-1$

$$
\begin{aligned}
& D_{h}^{(a)}(x)=\left\{\left[D_{h 1(01)} \exp \left(-2 \pi \mathrm{i} \Im k_{h 1(01)} x \sin \alpha\right)+D_{h 1(h 1)}\right.\right. \\
& \left.\times \exp \left(-2 \pi \mathrm{i} \Im k_{h 1(01)} x \sin \alpha\right) \exp \left(-2 \pi \mathrm{i} \Im k_{01} x \frac{\gamma_{h}}{\gamma_{h}^{\prime}} \sin \alpha\right)+D_{h 1(a)}\right\} \\
& \times \exp \left(2 \pi \mathrm{i} \Re k_{01(01)} x \gamma_{h}\right)+\left\{D_{h 2(01)} \exp \left(-2 \pi \mathrm{i} \Im k_{h 2(01)} x \sin \alpha\right)\right. \\
& \left.+D_{h 2(h 1)} \exp \left(-2 \pi \mathrm{i} \Im k_{h 2(01)} x \sin \alpha\right)\right\} \exp \left(-2 \pi \mathrm{i} \Im k_{01} x \frac{\gamma_{h}}{\gamma_{h}^{\prime}} \sin \alpha\right) \\
& \times \exp \left(2 \pi \mathrm{i} \Re k_{02(01)^{x}} x \gamma_{h}\right) .
\end{aligned}
$$

It is well known that the imaginary part of the wave vectors is treated as the component along the normal to the entrance surface: $\boldsymbol{n}$ in the region I and $\boldsymbol{f}$ in region II. Their values for both regions and all indices used can be found from the coordinates of the wave points

$$
\Im k_{0}=\Im\left[\gamma_{0}^{-1}\left(\xi_{0}+\frac{1}{2} K \chi_{0}\right)\right], \quad \Im k_{h}=\Im\left[\gamma_{h}^{-1}\left(\xi_{h}+\frac{1}{2} K \chi_{0}\right)\right]
$$

and the real parts

$$
\Re k_{0}=\Re\left(K+\xi_{0}+\frac{1}{2} K \chi_{0}\right), \quad \Re k_{h}=\Re\left(K+\xi_{h}+\frac{1}{2} K \chi_{0}\right) .
$$

In order to obtain better correspondence to the experiment, we took into account the divergence of the probe beam, by summing at least 60 components for different angles of incidence weighted by an appropriate rocking curve.

\section{Experimental}

The synchrotron experiments were performed at the station 7.6 of the S.E.R.C. Daresbury Laboratory storage ring. Owing to the $80 \mathrm{~m}$ distance of the experimental arrangement from the tangent point of the synchrotron source it was possible to accept a much worse matching from the reflections on the monochromator and investigated diamond. We used there the 511 reflection from a (111)-oriented silicon monochromator to match the 311 reflection from the diamond slab. The arrangement selected $1.54 \AA$ radiation, close to $\mathrm{Cu} \cdot K_{\alpha_{1}}$. The topographs were exposed in series with $0.5^{\prime \prime}$ angular adjustment to the sample between exposures. 


\section{Correspondence of experimental and theoretical images}

The $511_{\mathrm{Si}},-311_{\diamond}$ synchrotron double-crystal arrangement with $1.54 \AA$ radiation provided stacking faults images with interference fringes. The fringes were observed only for angular positions on the tails of the rocking curve; they become much less spaced further from the peak. The appearance of the fringes is shown in Fig. 2, showing their images in four angular settings - two on each tail of the rocking curve.
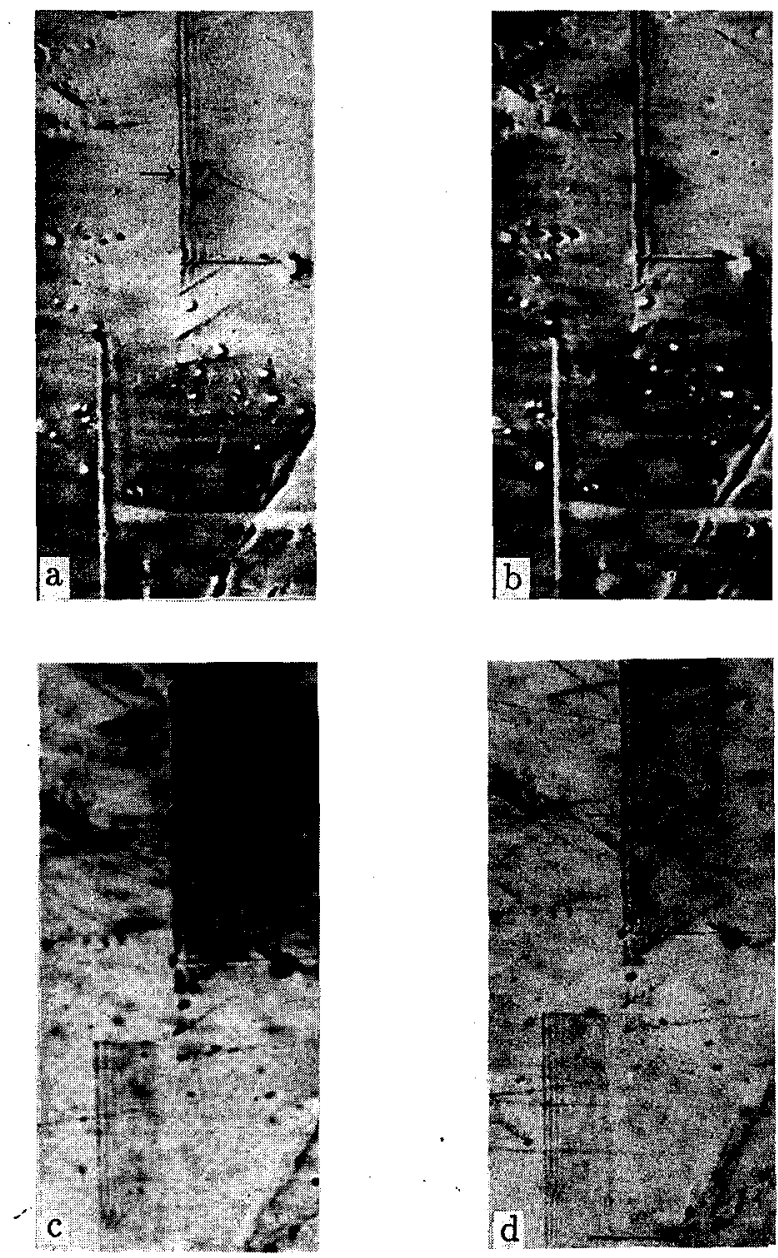

Fig. 2. The series of four double-crystal Bragg-Laue images of a stacking fault, taken with the $511_{\mathrm{Si}},-113_{\text {。 }}$ synchrotron arrangement with $1.54 \AA$ radiation. The topographs are ordered in increasing angle of incidence, (a) and (b) on the low-angle tail and (c) on (d) the high-angle tail of the rocking curve. Projection of the diffraction vector, in each case, is horizontal and pointing to the left. Image width $=400 \mu \mathrm{m}$. 

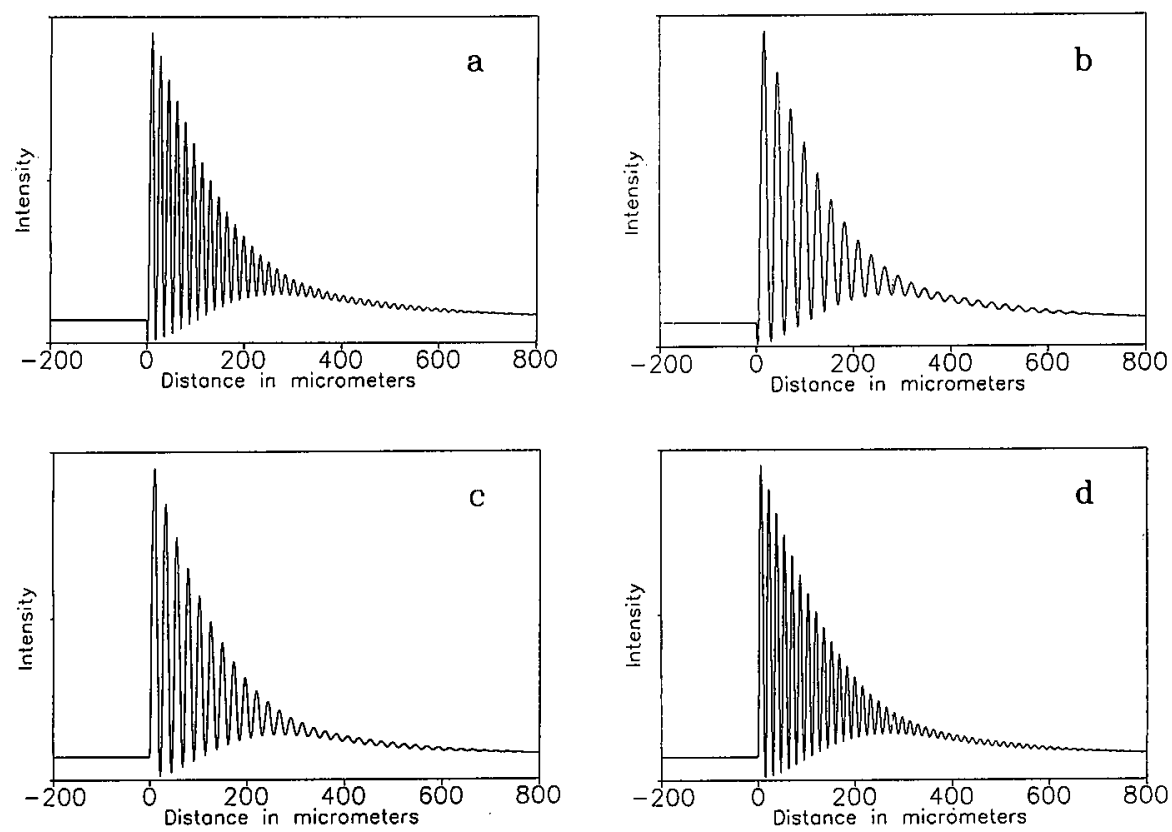

Fig. 3. The theoretical intensity distributions corresponding to the experimental images shown in Figs. 2a to $2 d$ assuming an intrinsic type of stacking fault (which more closely resembles the experimental images than the extrinsic type) and the $0.3^{\prime \prime}$ divergence of the incident beam.

Good visibility of interference fringes was due both to the spatial resolution being better than $1 \mu \mathrm{m}$ and to the very good combination of small incident beam divergence (of the order of $0.2^{\prime \prime}$ ) with a relatively wide intrinsic rocking curve (of $4.6^{\prime \prime}$ ) for the asymmetrical 311 diamond reflection. The relatively wide rocking curve had the effect of decreasing the influence of inclusions and other defects, thus causing the crystal to behave more like a perfect one.

The theoretical intensity distributions for intrinsic type of stacking fault corresponding to three of the experimental Bragg-Laue images shown in Fig. 2 are presented in Fig. 3. fault In these figures we assumed $0.3^{\prime \prime}$ divergence of the incident beam. The different types of stacking fault cause a small difference in the theoretical image close to the outcrop of the stacking fault (giving a difference in the starting phase of oscillations), and the image is different on the two sides of the rocking curve. Assuming of the extrinsic stacking fault approximately reverses the situation on the two slopes of the rocking curve. This features provide the possibility of identification of stacking fault type. The present images seem to. confirm that the stacking faults are of intrinsic type (see the detail marked by an arrow in Fig. 2).

The diffusion of the further fringes and the increasing of the background between fringes due to taking into account the beam divergence is very well visible in the experimental topographs. 


\section{Conclusions}

The Bragg-case images of stacking faults exhibiting distinct interference fringes were obtained in a slab cut from synthetic diamond using double-crystal topographic methods with a synchrotron X-ray source. The best visibility of fringes was due both to the very good spatial resolution and to the good combination of small divergence of the incident beam and the relatively wide rocking curve.

The fringe patterns were strongly dependent on the angular position on the rocking curve. They were invisible in the peak regions and became more closely spaced further from the peak.

The application of plane-wave theory for the diffraction from stacking faults was also discussed. We obtained a reasonably good correspondence between theoretical and experimental patterns, taking into account the finite divergence of the incident beam.

The starting sequence of the theoretical images close to the stacking fault outcrop had been found to depend on the type of the stacking fault, and this sequence was also dependent on the side of the rocking curve. This provided the possibility of identification of the stacking fault type.

\section{Acknowledgements}

The authors are greatly indebted to the Science and Engineering Research Council and to De Beers Industrial Diamond Division (Pty) for financial support. They would also like to thank Prof. A.R. Lang F.R.S (University of Bristol) for his helpful advice and to Mr. R. Waggett for taking part in some of the experiments.

\section{References}

[1] N. Kato, K. Usami, T. Katagawa, Adv. X-Ray Analysis 10, 46 (1967).

[2] A. Authier, Phys. Status Solidi 27, 77 (1968).

[3] S.-S. Jiang, A.R. Lang, Proc. R. Soc. Lond. A 388, 249 (1983).

[4] N. Kato, in X-Ray Diffraction, by L.V. Azároff, R. Kaplov, N. Kato, R.J. Weiss, A.J.C. Wilson, R.A. Young, Mc Graw-Hill, New York 1974, Chs. 3-5, p. 176.

[5] G. Kowalski, A.R. Lang, A.P.W. Makepeace, M. Moore, J. Appl. Crystallogr. 22, 410 (1989).

[6] H.M. Strong, R.H. Wentorf, Jr., Nalurwissenschaften 59, 1 (1972).

[i] A. Authier, Acta Crystallogr. A 42, 414 (1986). 